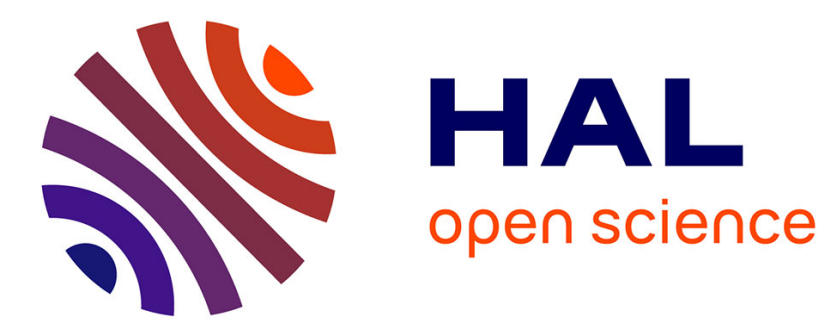

\title{
Ce qui ne cesse pas de ne pas commencer
}

\author{
Alain Vanier
}

\section{To cite this version:}

Alain Vanier. Ce qui ne cesse pas de ne pas commencer: D'un commencement sans origine. Cliniques méditerranéennes, 2002, Psychopathologie du travail, 2 (66), pp.145 - 153. 10.3917/cm.066.0145. hal-01522228

\section{HAL Id: hal-01522228 \\ https://hal.science/hal-01522228}

Submitted on 6 Jun 2017

HAL is a multi-disciplinary open access archive for the deposit and dissemination of scientific research documents, whether they are published or not. The documents may come from teaching and research institutions in France or abroad, or from public or private research centers.
L'archive ouverte pluridisciplinaire HAL, est destinée au dépôt et à la diffusion de documents scientifiques de niveau recherche, publiés ou non, émanant des établissements d'enseignement et de recherche français ou étrangers, des laboratoires publics ou privés. 


\section{Alain Vanier}

\section{Ce qui ne cesse pas de ne pas commencer \\ D'un commencement sans origine}

Ce qui est premier en date, primitif, est un des points cruciaux de la psychanalyse. Ce qui débute - terme qui a d'abord signifié « déplacer » - ce qui commence ( « initie ») se confond-il avec l'origine ( « l'ancêtre ${ }^{1}$ »), avec ce qui fait origine ? La conception de cette origine est paradoxale car, d'une part, elle n'est pas articulable en soi et, en même temps, elle est le point d'appui nécessaire à toute autre articulation. Pourquoi, pour Freud, cette dimension a-t-elle besoin de se trouver située, cernée voire figurée, pourquoi est-elle décisive pour la psychanalyse qui ne peut la laisser, comme dans d'autres domaines, insituable ou indéterminée, en tout cas contingente pour produire une théorie?

Ce ne serait pas forcer les choses que de dire que la psychanalyse est en elle-même une expérience et une théorie du temps, bien plus qu'une théorie produisant des conceptions du temps. Cette question elle-même est reprise à toutes les étapes de l'œuvre de Freud, jusqu'à un propos tardif rapporté par Marie Bonaparte où Freud identifie le temps avec l'attention 2. Mais il y a aussi la façon dont le temps apparaît divisé dans l'expérience sous des espèces contradictoires. D'emblée la conception du trauma et son statut

Alain Vanier, psychanalyste, professeur de psychopathologie clinique à l'université de Paris VII, Denis Diderot.

1. A. Rey, 1992.

2. M. Bonaparte, 1939. «L'attention que nous portons aux choses serait due à des investissements rapides, mais successifs, sortes de quanta émané du moi. Notre perception interne n'en ferait qu'ensuite une continuité, et ce sera là, projeté au-dehors, notre prototype du temps. » Freud ajoute que ces « quanta d'investissements primitifs sont ensuite rétablis dans le temps par l'homme, avec sa fragmentation du temps mesurable. Il resterait de tout ceci que attention = perception = temps. » 
comme événement psychique se trouvent pensés par Freud dans une perspective temporelle singulière, où l'on peut dire que c'est la période de latence, l'entre-deux temps qui devient essentiel. Ce n'est en effet que dans l'après-coup, après un temps où rien ne se manifeste, au moment de la puberté que le statut de l'événement premier, du moment traumatique trouve à s'inscrire. Si le premier temps ne prend valeur traumatique qu'à partir du moment où le second rétroagit sur lui, la primauté devient problématique, et, pour la psychanalyse, il est conséquent de constater que l'événement psychique ne coïncide jamais avec lui-même.

Cette rétroaction implique une dimension anticipatrice du premier temps. En effet, n'importe quel événement ne peut le constituer. Lacan a repéré ces mouvements d'anticipation et de rétroaction comme propres aux régulations de la chaîne signifiante. De la même manière, il propose un modèle de "triangle préœdipien ", où, dès sa venue au monde, son entrée dans le champ du collectif et de la culture, l'enfant est inscrit dans un rapport non seulement à la mère mais aussi d'ores et déjà au phallus, comme organisant, en amont de son origine, le monde de celle-ci. Cette anticipation se trouve, me semble-t-il, vérifiée dans la pratique des analystes d'enfants. Si l'on considère la rencontre de Dick avec Melanie Klein, cet avant-coup de la structure, cette forme anticipée est ce qui fonde et permet l'efficacité de l'énoncé du mythe œdipien, à partir de la supposition de savoir posée par $l^{\prime}$ analyste dans l'enfant ${ }^{3}$. Dire que le trauma n'est trauma qu'à partir du deuxième temps et que c'est du deuxième temps que nous pourrions situer le premier, peut paraître trivial. Cette anticipation se repère dans la relation entre la mère et son enfant, dans le savoir qu' elle lui suppose, dont elle fait "l'hypothèse 4 ", et qui la fait, peut-on dire, le lieu d'une anticipation du sujet qu'elle porte et soutient de sa supposition ${ }^{5}$. Dès lors, ce premier temps, orienté vers le suivant, en attente du suivant, est aussi bien le second d'un temps qui lui serait postérieur.

Sur un autre versant, il y a la psychogenèse. Celle dont l'établissement, l'énoncé dans l'œuvre freudienne n'arrive que tardivement. L'idée d'une temporalité conçue comme développement, l'élaboration des stades, a sa source dans la théorie de l'évolution, et ces étapes du développement sont une sorte de théorie de l'évolution de l'individu, où " l'ontogenèse peut être considérée comme une répétition de la phylogenèse ${ }^{6} »$. Mais, paradoxale-

3. M. Klein, 1930. Sur la question de la position de l'analyste dans ces cures, voir A. Vanier, 1993. 4. Voir J. Bergès, G. Balbo, 1998. Ce qui rend compte de cette «préparation à comprendre » dont parle S. Freud, 1918b, p. 117.

5. Voir A. Vanier, 1989.

6. S. Freud, $1905 d$, p. 29. 
ment, l'idée de l'évolution, bien que contredisant les grands mythes religieux de l'humanité, ne conforte-t-elle pas une perspective temporelle qu'elle doit au monothéisme et qui singulièrement la fonde et la permet ?

Dans un article de 1957, Winnicott distingue ce qui est profond (deep) au sens analytique de ce qui est primitif (early) dans le sens du développement du nourrisson. Acceptons ces qualifications pour simplement relever que ce qui est en jeu dans le mouvement de la cure, ce que Winnicott appelle leprofond, et qui veut dire pour lui aller en profondeur " dans le fantasme inconscient ou dans la réalité psychique du patient » n'est pas la même chose que ce qui est repérable en terme de développement et qui est produit par l'observation des nourrissons. Winnicott en donne un exemple. Pour lui la dimension nécessaire de l'environnement au tout premier temps n'est pas remémorable dans l'analyse. Cette « conception de l'environnement doit être ajoutée par l'analyste » au cours de la cure. Car « dans l'analyse, cet environnement est sous-entendu, mais le patient ne peut le communiquer parce qu'il n'en a jamais pris conscience $^{7} »$. Ainsi, il remarque concernant le holding que "sans environnement, le nourrisson ne cesserait de tomber (would fall infinitely)». Il ajoute que le nourrisson qui est normalement porté n'a pas conscience « qu'on ne cesse de l'empêcher de tomber». C'est seulement le défaut de ce portage qui peut apparaître dans la cure comme sentiment de chute évoqué par le patient mais, souligne Winnicott, «il ne peut jamais dire qu'il a été porté pendant cette première étape du développement ». Il n’y a néanmoins, bien que ce texte se termine par un appel à une collaboration entre les analystes et les psychologues du développement, aucune complémentarité véritable, car il y a « beaucoup plus dans la nature humaine que ce qui peut être observé directement ».

La cure analytique bouleverse les conceptions traditionnelles de la temporalité. Peut-on d'ailleurs rigoureusement y distinguer un temps, ou n'y at-il aucune articulation entre le sentiment du temps qui s'écoule et la face intemporelle qui habite toute subjectivité ? Dans la cure de l'Homme aux Loups, Freud rencontre une limite à la remémoration, à ce mouvement de l'avenir vers le passé qui oriente le traitement. Le trauma initial qu'a rencontré le patient n'a pu être mis à jour par la mémoire. Il a fallu que Freud le construise. La scène traumatique aurait eu lieu quand le patient était âgé d'un an et demi. Freud discutera très longuement la question de la réalité de cette scène, de ce coït parental observé lors d'une sieste. Il faut remarquer ici que, quand bien même le patient se la serait remémorée, ce ne serait pas plus une garantie de sa réalité. Dans le cours d'une analyse les sujets sont amenés

7. D. W. Winnicott, 1957, p. 78. 
à faire surgir du passé des souvenirs. Or, ceux-ci ne sont pas pour autant la marque d'une vérité factuelle, un gage de véracité. Ils sont, le plus souvent, faits d'éléments advenus à des périodes différentes, recomposés, ce que Freud nomme des souvenirs-écrans. Ici, concernant cette scène primitive, Freud maintient qu'elle est de l'ordre de la réalité, et, malgré ses doutes, il est pour lui nécessaire de penser qu'elle a effectivement eu lieu ${ }^{8}$. Et ceci est $d^{\prime}$ autant plus remarquable qu'elle n'est pas attestée par la remémoration. Mais elle a eu lieu, car elle a un lieu.

À propos de cet événement traumatique, Lacan propose d'utiliser le terme de Prägung. C'est l'empreinte, mais c'est aussi la frappe au sens où l'on frappe une monnaie. "La Prägung n'a pas été intégrée au système verbalisé du sujet » (1953-1954). Qu'est-ce qui se passe deux ans plus tard ? « Le sujet apprend à intégrer les événements de sa vie dans une loi, dans un champ de signification symbolique, dans un champ humain universalisant de significations. C'est pourquoi au moins à cette date, cette névrose infantile est exactement la même chose qu'une psychanalyse. » Car la psychanalyse effectue la réintégration du passé. Ce qui pourrait nous porter à dire que, d'une certaine manière, le trauma peut avoir lieu dans l'analyse. La névrose infantile apparaît ainsi comme réponse du sujet face à ce qui apparaît comme littéralement traumatique : le désir énigmatique de l'Autre, et la question de sa jouissance. Dans certaines cures d'enfant, le rôle de l'analyste n'est-il pas alors de donner un coup de pouce au processus en cours, à l'élaboration fantasmatique qui est en train de se produire? Ainsi s'expliquerait, peut-être, la place prépondérante que Melanie Klein a donnée à la vie fantasmatique précoce à partir d'analyses d'enfants d'environ deux ou trois ans.

Le trauma, donc, intervient après-coup. « À ce moment-là, quelque chose se détache du sujet dans le monde symbolique même qu'il est en train d'intégrer. Désormais, cela ne sera plus quelque chose du sujet. Le sujet ne le parlera plus, ne l'intégrera plus. Néanmoins, ça restera là, quelque part, parlé, si l'on peut dire, par quelque chose dont le sujet n'a pas la maîtrise. Ce sera le premier noyau de ce que l'on appelle par la suite ses symptômes. »

Freud insiste pour accorder crédit à sa construction, mais en même temps il souligne qu'il n'est pas essentiel de savoir si, chez l'Homme aux loups, il s'agit d'un fantasme ou d'un vécu réel. Ces scènes peuvent être " une possession héritée, un héritage phylogénétique, mais elles peuvent être aussi bien l'acquis d'un vécu personnel ». Il ajoute : «L'enfant [...] comble les lacunes de la vérité individuelle avec de la vérité préhistorique, il remplace sa propre expérience par celle de ses ancêtres » (1918b, p. 399-400). La scène 
première ne peut donc s'atteindre par la remémoration parce qu'elle n'est pas symbolisée. Elle ne prend place dans les rets du symbolique qu'à partir du deuxième temps. Le premier temps du traumatisme est donc un temps préhistorique. Freud ici fait référence aux religions, à la fonction du père et à celle de Dieu. Il évoque Totem et tabou et la fonction originaire du père, avec ce que la scène primitive présente de sa jouissance qui conduit à situer le père dans la préhistoire (1912-1913). Totem et tabou en est le mythe : avant l'histoire, une horde menée par un père demi-animal qui possède toutes les femmes et en prive les fils. Les fils ourdissent un complot contre le père et le tuent. Mais pour pouvoir jouir des femmes, ces frères ne manqueraient pas de continuer à s'entre-tuer. Pour arrêter ce processus, ils concluent entre eux un pacte qui totémise le père et impose le tabou sur les mères. Cette Loi est bien celle du Père, il ne s'agit pas de consensus, car il y faut son Nom. Le résultat de l'opération est que le père mort, le totem, impose sa loi d'une façon encore plus efficace que lorsqu'il était vivant. Le père apparaît ainsi, selon le mot de Lacan, comme gardien de la jouissance. L'origine de l'histoire est donc traumatique. Elle s'organise autour d'un élément non historisable. Si l'événement traumatique lui-même ne peut apparaître dans la remémoration, ce n'est pas par un défaut propre à cette analyse-là, mais c'est un fait de structure. La fonction initiale que Lacan nommera Nom-du-Père est l'origine de l'histoire, elle est aussi le fondement d'une dimension du temps, du temps pour le sujet. Ainsi cette remémoration, ce mouvement régressif propre au traitement analytique trouve sa limite. Nous remontons selon des signifiants mais non selon le fil temporel qui a constitué la trame des jours successifs de notre vie. Cette remémoration a elle-même une limite et Freud évoque la notion d'entropie. À la fin de l'Homme aux loups, il propose l'hérédité comme cause, ainsi se transmet l'Edipe comme l'un des « dépôts de l'histoire culturelle humaine ", présent dans le langage lui-même, et transmis avec lui. Le trauma est ainsi, fondamentalement, rencontre avec le langage.

La jouissance du père de la horde, qui possède toutes les femmes, est sans limite. Si l'effet de Totem et tabou est de proposer un mythe rendant compte de la mise en place de ce que Lacan appellera le Nom-du-Père, notons néanmoins que cette fonction ne peut se passer du père réel, d'une instance agent de séparation. Dès l'origine, Freud rencontre le père, qui parcourra toute son œuvre, qu'il formule d'abord avec la théorie de la séduction : fondamentalement, la sexualité est " hétéro », elle vient de l'Autre. Le complexe d'CEdipe, Totem et tabou, le Moïse sont autant de reprises de cette question, dans un mouvement de dégagement, tâche poursuivie par Lacan. Mais ce père réel situé par Lacan comme agent de la castration, n'a-t-il comme fonction que d'être celui qui relaie le père symbolique et en transmet la dimension? Ce serait réduire le père réel au père de la réalité. Car il est 
aussi affleurement de l'origine jouissante et infinie du père de la préhistoire. Il est aussi celui qui, dans la réalité, jouit réellement de la mère, de celle qui subsume toute femme, qui est en quelque sorte, un temps, toute femme pour le sujet. Mais cette rencontre se figure fantasmatiquement car elle n'est qu'en défaut, là où elle pourrait constituer une origine. La Prägung évoquée par Lacan suppose le creux qui donnera son relief à la pièce, elle est ce réel, plus tardivement défini comme manque du rapport sexuel, «à savoir une relation définissable comme telle entre le signe du mâle et celui de la femelle ». Ici se projette la scène primitive car « il n'y a pas d'acte sexuel au sens où cet acte serait celui d'un juste rapport, et [...] inversement, il n'y a que l'acte sexuel, au sens où il n'y a que l'acte pour faire le rapport » (1968-1969).

Ainsi peut se comprendre cette double face qui lie le père au nom et que Lacan distinguera dans les dernières années de son enseignement. D'une part le Nom-du-père, mais aussi le père qui nomme, nomination pas sans jouissance, au moins inférée (1975-1976). L'hallucination verbale dans la clinique des psychoses en témoigne quand le Nom-du-Père est forclos. La théorie du langage de Benjamin et sa conception de l'origine ne sont pas sans intérêt et résonance pour les questions qui nous occupent. Si « l'homme est celui qui parle ", pour autant " ni dans la nature animée ni dans la nature inanimée, il n'existe événement ni chose qui, d'une certaine façon, n'ait part au langage » (1916). L'essence linguistique présente « des différences de degrés » qui correspondent à un «étagement » de l'être. Reprenant la Genèse, non dans un but d'exégèse biblique, mais pour « explorer ce que nous présente la Bible quant à la nature même du langage ", il remarque le rythme ternaire de la création de la nature : Que soit fait - Il fit (créa) - Il nomma. L'acte créateur " commence avec la toute-puissance créatrice du langage, et pour finir le langage s'incorpore en quelque sorte le créé, il le dénomme ». En Dieu seulement, le langage est savoir et nomination en même temps. Ce degré est celui du langage de noms. À un autre niveau, les choses de la nature ainsi créées demeurent silencieuses et sont traversées par « un langage muet et sans nom, résidu de ce verbe créateur et divin ». L'homme adamique est le seul qui n'ait pas été créé à partir du verbe, mais il est aussi le seul qui connaît le langage créé par Dieu, bien qu'il soit, à ce niveau, "vidé de son actualité », c'est-à-dire que, chez lui, " ce pouvoir créateur est devenu connaissance ». En effet, si la langue adamique est celle du " parfait savoir » qui est sa capacité traductrice, elle n'est plus créatrice du monde maintenant muet des choses. Un degré de plus et c'est le monde d'après Babel, celui de l'éparpillement des langues, conséquence pour Benjamin du péché originel, responsable d'une disjonction d'avec le savoir : « En quittant le pur langage $\mathrm{du}$ nom, l'homme fait du langage un moyen (une connaissance qui ne lui convient pas), par là même aussi, en tout cas, pour une part, un simple 
signe. » Faute de pouvoir nommer les choses de leur nom propre, elles sont dans la langue des hommes "surdénommées ». Le mot est alors connaissance du dehors, littéralement impropre, il est séparé de la chose, c'est un nom déchu, dans le moment même de la chute du paradis originaire. Il manifeste l'inadéquation du signifiant et de la chose. Ce qu'avance Benjamin figure remarquablement ce mouvement incessant des signifiants, celui de la régression propre à l'analyse, comme tentative de reconquête, de retrouvaille de cette part à jamais perdue et en fait inatteignable. Ce qui est originairement perdu est le "souffle », la voix et l'énonciation divines, l'acte créateur de nomination lui-même, sa jouissance - acte et verbe qui furent au commencement.

Cette perte fondamentale, cette chute qui pourrait être infinie selon Winnicott, cet objet qui choit, peut se lire avec Lacan, pour revenir au holding, comme figure de l'enfant en tant qu'objet $a$. À l'origine, il y a aussi pour le sujet l'objet qu'il aura été pour l'Autre. Dans la séance unique de son séminaire de 1963, Les Noms du Père, Lacan souligne comment il est nécessaire pour Freud de supposer à l'origine de la conjonction de la loi et du désir, la jouissance pure du père comme primordiale. Évoquant le sacrifice d'Abraham, Lacan reprend le commentaire du Pirke Avot (Traité des Pères) où il est dit que le bélier qui vient se substituer à Isaac dans le sacrifice est le bélier primordial. «Il était là - hassé mimé berechit - dès les six jours de la création, ce qui le désigne pour ce qu'il est : un Elohim. Ce n'est pas celui dont le nom est imprononçable, mais tous les Elohim. » Lacan peut ainsi conclure : « $\mathrm{Ce}$ qu'Elohim désigne pour sacrifice à Abraham à la place d'Isaac c'est son ancêtre, le dieu de sa race. Ici se marque le tranchant entre la jouissance de Dieu et ce qui d'une tradition le désigne comme désir, désir de quelque chose dont il s'agit de provoquer la chute c'est : l'origine biologique [...]. Quelque chose se manifeste qui, comme étant le désir, met essentiellement en valeur cette béance qui sépare la jouissance du désir et le symbole en est [...] la circoncision, signe de l'alliance du peuple avec celui qui l'a élu, la circoncision désigne ce petit morceau de chair tranché à l'énigme duquel je vous avais amenés par quelques hiéroglyphes, ce petit $a »$ (1963). Ce qui vient ici se substituer au sacrifice d'enfant, $c^{\prime}$ est donc le père originaire, bestial, qu'il s'agit une nouvelle fois de sacrifier. Mais parce qu'il vient à cette place, il incarne aussi cet enfant, notre ancêtre, cet être si près de la nature, à la fois objet $a$ luimême et inscriptible comme sujet dans la communauté seulement par la séparation d'avec cet objet qu'il était. Dans cette perspective le statut même de l'infantile, son inlassable action jouissante est sa manifestation, pour une part, dans, peut-on dire, " un présent qui n'est pas un passage, mais se tient immobile sur le seuil du temps » (W. Benjamin cité par G. Agamben, 1978). 
Ce qui est premier en date est impensable sans la datation. L'enfant, comme le père, dont Dieu est l'une des faces, sont figurations du primitif ( primitiv) d'où l'usage régulier de ce terme chez Freud aussi bien pour les enfants que pour les peuples dits primitifs ${ }^{9}$. Or, il y a quelque chose de l'origine qui ne parvient pas à être premier, mais qui en témoigne toujours au présent, qui fait retour éternellement. Dans la reprise du deuxième temps du trauma, tout comme dans la conception des stades, il y a toujours un reste qui excède ce retour, ou qui fait de ce retour un excès, qui fait qu'il n'est pas seulement retour mais présence, il est là ${ }^{10}$. « L'origine, bien qu'étant une catégorie tout à fait historique, n'a pourtant rien à voir avec la genèse des choses. L'origine ne désigne pas le devenir de ce qui est né, mais bien ce qui est en train de naître dans le devenir et le déclin. L'origine est un tourbillon dans le fleuve du devenir, et elle entraîne dans son rythme la matière de ce qui est en train d'apparaître » (Benjamin, 1928). Origine non primaire, elle est cette part retranchée que le langage ne cesse de traiter, elle constitue le mobile du sujet et du lien social, ce qui le meut, car ce qui a permis de commencer est aussi ce qui ne cesse pas de ne pas commencer.

\section{BIBLIOGRAPHIE}

Agamben, G. 1978. Enfance et histoire, trad. Y. Hersant, Paris, Payot, 1989.

Benjamin, W. 1916. «Sur le langage en général et sur le langage humain », dans Mythe et violence, trad. M. de Gandillac, Paris, Denoël, 1971.

Benjamin, W. 1928. Origine du drame baroque allemand, trad. S. Muller, Paris, Flammarion, 1985.

BerGÈs, J. ; BALBo, G. 1998. Jeu des places de la mère et l'enfant. Essai sur le transitivisme, Toulouse, Érès.

BONAPARTE, M. 1939. "L'Inconscient et le temps », dans Revue française de psychanalyse, XI, I, 1939, repris dans Chronos, Éros, Thanatos, Londres, Imago Publishing Co, 1952.

DelRieu, A. 1997. Sigmund Freud, index thématique, Paris, Anthropos-Economica.

FREUD, S. 1905. Trois essais sur la théorie sexuelle, trad. P. Koeppel, Paris, Gallimard, 1987.

FREUD, S. 1912-1913. Totem et tabou, quelques concordances entre la vie psychique des sauvages et celle des névrosés, trad. M. Weber, Paris, Gallimard, 1993.

FreUd, S. 1918b. «Extraits de l'histoire d'une névrose infantile ». In Cinq psychanalyses, trad. M. Bonaparte et R. Loewenstein, Paris, PUF, 1954.

9. Voir A. Delrieu, Sigmund Freud, index thématique, Paris, Anthropos-Economica, 1997.

10. Ce que relève Winnicott à propos de l'adolescence comme mettant en jeu avant tout « la mort de quelqu'un ». 
Gori, R., Hoffmann, C. 1999. La science au risque de la psychanalyse. Essai sur la propagande scientifique, Toulouse, Érès, 1999.

KLEIN, M. 1930. «L'importance de la formation du symbole dans le développement du moi », dans Essais de psychanalyse, trad. M. Derrida, Paris, Payot, 1968.

LACAN, J. 1953-1954. Les écrits techniques de Freud, Le séminaire, livre I, texte établi par J.-A. Miller, Paris, Le Seuil, 1975.

LaCAN, J. 1963. Les Noms du Père, Inédit, 20 novembre.

LaCAN, J. 1968-1969. D'un Autre à l'autre, Le Séminaire, livre XVI, inédit.

LACAN, J. 1975-1976. Le sinthome, Le séminaire, livre XXIII, inédit.

ReY, A. (dir.) 1992. Dictionnaire historique de la langue française, 2 vol., Paris, Le Robert. VANIER, A. 1989. «Quelques remarques à propos des mères psychotiques et leur bébé ", Communication au IVe Congrès mondial de la WAIPAD (World Association for Infant Psychiatry and Allied Disciplines), Lugano (Suisse), 20-24 septembre 1989, inédit.

VANIER, A. 1993. « Autisme et théorie », dans Hommage à Frances Tustin, Saint-Andréde-Cruzières, Éd. Audit,.

WinnicotT, D.W. 1957. " Contribution de l'observation directe des enfants à la psychanalyse ", dans Processus de maturation chez l'enfant, trad. J. Kalmanovitch, Paris, Payot.

Traité des Pères (Pirqé Avot) et commentaires, trad. E. Smilévitch, Paris, Verdier, 1990.

Résumé

Toute une série de termes désignant le commencement insistent dans les écrits psychanalytiques depuis Freud. Leur variété, les contradictions et les paradoxes théoriques qu'ils provoquent et qui ne sont pas sans conséquences pour la pratique sont autant d'indices de la complexité du problème de la temporalité en psychanalyse.

Mots clés

Développement, enfance, jouissance, père, primitif, temps, traumatisme.

\section{THAT WHICH CEASELESSLY FAILS TO COMMENCE}

\section{Summary}

A whole series of terms describing the commencement have been emphasised in psychoanalytical writings since Freud. Their variety and the contradictions and theoretical paradoxes they cause and that are not free of consequences for practice provide so many clues to the complexity of the problem of temporality in psychoanalysis.

Key words

Development, childhood, enjoyment, father, primitive, time, traumatism. 